\title{
Incorporación y apropiación de las TIC en los procesos de enseñanza y aprendizaje en el nivel de educación media
}

\author{
Incorporation and appropriation of TIC in the teaching and learning processes at \\ the level of middle education \\ R. R. Alvarez-Sampayo iD ; R. R. Sarmiento Guevara ; T.R. Amaya-De Armas iD \\ DOI: https://doi.org/10.22517/23447214.24191 \\ Artículo de investigación científica y tecnológica
}

\begin{abstract}
Resumen - Este trabajo tiene por objetivo proponer estrategias de incorporación y apropiación de las TIC en los procesos de enseñanza y aprendizaje en el nivel de educación media académica o secundaria alta, a partir de la identificación de los niveles de uso de las TIC por parte de los docentes, el análisis del desarrollo de competencias TIC en ellos y la proyección de acciones institucionales que permitan un aprovechamiento efectivo de los recursos tecnológicos existentes en los establecimientos educativos. La investigación se enmarca como estudio de casos, desde un enfoque cualitativo, complementado con técnicas y análisis de información cuantitativa. Se trabajó con 270 estudiantes, 24 docentes, 5 directivos y 7 acudientes o padres de familia, en dos instituciones educativas. Existen acuerdos altamente significativos entre establecimientos educativos, que evidencian una moderada incorporación y apropiación de las TIC por los docentes, escasa creación de ambientes innovadores utilizando las TIC e insuficiente infraestructura tecnológica. Se destacan levemente las competencias: tecnológica, comunicativa y de gestión. Se concluye que las TIC son empleadas en su mayoría desde la pedagogía tradicional, con un nivel de exploración.
\end{abstract}

Palabras claves-Incorporación y Apropiación de TIC, Enseñanza y Aprendizaje, Niveles de Competencias TIC.

\begin{abstract}
The objective of this work is to propose strategies of incorporation and appropriation of ICT in the teaching and learning processes at the level of academic middle education or high secondary, based on the identification of the levels of use of ICT by teachers, the analysis the development of ICT skills in them and the projection of institutional actions that allow an effective use of existing technological resources in educational establishments. The research is framed as a case study, from a qualitative approach, complemented with techniques and analysis of quantitative information. We worked with 270 students, 24 teachers, 5 managers and 7 caregivers or parents, in two educational institutions. There are highly significant agreements between schools, which show a moderate incorporation and appropriation of ICT by teachers, the scarce creation ofinnovative environments with ICT and insufficient technological infrastructure. The competencies are slightly highlighted: technological, communicative and management. It is concluded that ICT are mostly used from traditional pedagogy, with a level of exploration.
\end{abstract}

This manuscript was sent on May 28, 2020 and accepted on February 05 , 2021.
Index Terms-Incorporation and Appropriation of ICT, Teaching and Learning, ICT Skills Levels.

\section{INTRODUCCIÓN}

Gxiste una dinámica de promoción y priorización de las ¿TIC en función del desarrollo, en donde lo educativo es esencial y se prioriza la construcción de la sociedad de la información integradora, en la cual la incorporación de las TIC en la educación es un elemento estratégico, que implica la erradicación del analfabetismo digital y la definición de políticas para garantizar su integración en todos los niveles educativos. Esta integración implica una intervención en: los planes de estudio, la formación de docentes, lo administrativo en las instituciones, y favorecer el proceso de aprendizaje de los estudiantes en cuanto a conocimientos y capacidades en el aprovechamiento de las tecnologías, incluyendo el uso de la información de forma analítica, creativa e innovadora, de caras al intercambio de experiencias e inserción en la sociedad de la información [1].

En la actualidad los países de América Latina y el Caribe han dedicado esfuerzos y recursos para introducir las TIC en todos los sectores de la sociedad. Uno de los ámbitos importantes en el cual han centrado gran parte de sus energías es la educación, estudiando el impacto de las TIC en los sistemas educativos, especialmente en los procesos de enseñanza y de aprendizaje en los ámbitos privado y público, buscando favorecer, el nivel de aprovechamiento por parte de los profesores y el desarrollo de software educativos en las escuelas públicas en pro del mejoramiento del desempeño de los estudiantes [2].

En Colombia las competencias que deben desarrollar los docentes de cara a la innovación educativa con la implementación de TIC deben ser cinco: Tecnológicas, Comunicativas, Pedagógicas, De Gestión e Investigativa [3]. En este sentido, se han venido impulsando diferentes programas y proyectos sobre la integración de las TIC en las instituciones públicas, que han permitido avances significativos en cuanto a la reducción de la brecha digital, aunque con muchas deficiencias, pues se ha logrado alcanzar solo niveles bajos de uso y apropiación. 
Experiencias de investigación como las reportadas en el programa Computadores para Educar, en proyectos de aula del Amazonas, Bogotá Distrito Capital, Cundinamarca, Guainía, Meta y Vaupés, evidencian la utilización de las TIC como medios de información y comunicación, pero su uso en el aprendizaje se sitúa en un nivel bajo o de preintegración [4]. Por otro lado, se describen las creencias y los conocimientos acerca de las TIC y los propósitos de su uso en la enseñanza en un grupo de profesores universitarios, que manifiestan conocer las TIC, pero la mayoría aplican solo algunas de ellas [5].

A su vez, en instituciones de niveles básica y media de la ciudad de Bogotá, se han identificado prácticas de integración educativa de TIC, caracterizando buenas prácticas y realizando una propuesta de formación que posibilita la gestión del conocimiento y el desarrollo profesional docente. En donde se reconoce que las prácticas de integración de TIC surgen mayormente por iniciativas personales de los docentes y se queda limitado a la acción en el aula de clases, las cuales se alinean en pocas oportunidades con objetivos estratégicos y metas institucionales o de la nación. No existe una tendencia marcada a la innovación, aunque se están desarrollando experiencias que se pueden considerar novedosas. El programa de formación propuesto, pretende gestionar el conocimiento desde la sostenibilidad del desarrollo profesional de los docentes a través de las prácticas institucionales contextualizadas, la articulación de las experiencias con las tendencias educativas con TIC y la potenciación de las alianzas externas y trabajo colaborativo con pares [6].

Es claro que los anteriores resultados, son similares en muchas de las regiones y entes territoriales del país, en donde la preocupación ante el insuficiente aprovechamiento de las Tecnologías de la Información y Comunicación, es manifestada explícitamente por diferentes actores educativos.

Ante la situación de abastecimiento tecnológico que se viene dando en las instituciones educativas públicas de Colombia, como resultado de las políticas nacionales de formar ciudadanos digitales, es de gran pertinencia responder algunas preguntas claves que están en sintonía con las incertidumbres que se presentan entre sus docentes e incluso sus directivos, que muchas veces no saben qué hacer con todos esos recursos o cómo aprovecharlos pertinentemente. Por una parte, se encuentra la apatía y/o el analfabetismo tecnológico, por otra, la mínima capacitación o escaza profundización tecnológica de la mayoría de los docentes de las instituciones, que afecta la utilización de los diferentes medios que se encuentran al alcance.

Es una realidad la incidencia educativa que tienen las TIC, es también una situación real que se generan una gran cantidad de inquietudes, dudas, prevenciones y muy contados casos en donde se logran identificar actitudes proactivas a la hora de su implementación. Además, hay una serie de condicionamientos ambientales y en la infraestructura física y tecnológica que también inciden en la real utilización y aprovechamiento efectivo de las dichas tecnologías. Pero algo sustancial es el desconocimiento, y por ende la deficiente apertura y compromiso con respecto a las Competencias TIC para el desarrollo profesional docente.
Si no se integran pertinentemente las TIC en la práctica docente y los procesos educativos, no se impulsa la implementación de nuevas didácticas y no se potencia los enfoques pedagógicos asumidos por los docentes, lo que puede repercutir en la escasa generación de ambientes de aprendizaje que estimulen el interés y la motivación de los estudiantes. Se desaprovecha la construcción de conocimiento, la estimulación de la imaginación, la creatividad, y el sentido crítico, que pueden ser estimulados por el análisis simultaneo de diferentes representaciones dinámicas de un objeto estudiado. En este sentido, se escatima la diversidad de fuentes de información; y no se toman como aliados los elementos que posibilitan los diferentes ambientes computacionales, como medios para facilitar la comprensión científica y para permitir el aprendizaje multisensorial.

Este trabajo apunta al desarrollo de estrategias de incorporación y apropiación de las TIC en los procesos de enseñanza y aprendizaje en el nivel de educación media académica de dos instituciones educativas, a partir de la identificación de la realidad institucional en este aspecto fundamental y actual, además de la constatación de los niveles de desempeño reales. Atiende a la necesidad de mejorar la práctica docente y los procesos de enseñanza y aprendizaje, mediante el aprovechamiento pertinente de las TIC en función del desarrollo integral de los estudiantes y su proyección a una sociedad globalizada que se apoya en un amplio y dinámico progreso tecnológico. Con mucha más razón, si se tiene en cuenta el escaso uso de las TIC en la práctica profesional de los docentes, que incide en el bajo nivel de aprovechamiento de los diferentes recursos y medios tecnológicos que se encuentran a su alcance. El uso apropiado de las TIC, conllevaría a plantear políticas y acciones institucionales contextualizadas que impulsen de forma concreta $y$ comprometida su integración en la enseñanza para el mejoramiento continuo de la práctica docente.

A partir de la problemática planteada, se buscan proponer acciones estratégicas de incorporación y apropiación de las TIC en los procesos de enseñanza y aprendizaje que apunten a mejorar la práctica docente en el nivel de educación media o secundaria alta, en las instituciones educativas, a partir de la identificación de los niveles de uso y apropiación por parte de los educadores, el análisis de los momentos de competencias TIC en que se encuentran y la definición de líneas de acción contextualizadas.

\section{FUNDAMENTACIÓN TEÓRICA}

\section{A. Incorporación y apropiación de TIC en entornos educativos}

La dinámica de incorporación y apropiación es considerada sustancial en la actualidad por parte de muchos autores, que enfatizan la importancia de las nuevas tecnologías a nivel educativo. Por lo que "las TIC se convierten en un instrumento cada vez más indispensable en las instituciones educativas donde pueden realizar múltiples funcionalidades [7]".

Para una efectiva incorporación y gradual apropiación de las TIC, es necesario que se presenten algunas acciones alternativas y complementarias, entre las que se encuentra, la necesidad de un mayor acompañamiento y profundización en 
los usos reales de las herramientas tecnológicas en las actividades formativas en pro de la construcción del conocimiento, el aprendizaje autorregulado y el pensamiento crítico y autónomo [5]. Además es ineludible gestar entornos virtuales desde enfoques pedagógicos debidamente asumidos para permitir que las TIC coadyuven en la consolidación de aprendizajes colaborativos abiertos y flexibles [8]. Es por tanto, indispensable progresar en el uso de metodologías innovadoras con el aprovechamiento de recursos tecnológicos en el ejercicio educativo, superando la posición tradicionalista y de poca apertura a los cambios [9].

Así mismo, las diferentes concepciones y creencias de los docentes con respecto al proceso educativo y a la utilización de las tecnologías en el mismo, pueden estar directa o indirectamente relacionadas con una pertinente y eficaz incorporación y apropiación de las TIC en la práctica docente en el aula.

La gestión de las creencias en los profesores es una línea de trabajo imprescindible, dado que su intención y conciencia pedagógica de lo que hacen es el factor de influencia más directa en el aprovechamiento didáctico de la tecnología y del desarrollo educativo de la humanidad en su manifestación más global [10].

Implementar el programa educativo basado en las TIC mejora los resultados escolares y la motivación de los estudiantes. Para ello es importante, desarrollar experiencias aplicadas a distintas áreas del saber, formar y capacitar a los docentes en la universidad en la creación de materiales didácticos multimedia para el desempeño en el aula. Esto permitiría acercar las tecnologías a la labor docente, sin verlas como algo complejo, considerando sus utilidades y ventajas para el rendimiento y la motivación del alumnado, mejorando el sistema educativo [11]. El docente es clave para superar el tecnocentismo, adaptando las opciones didácticas al aprendizaje de los estudiantes, trabajando en equipo y con una dinámica reflexiva que ayude a cambiar positivamente su práctica educativa [12].

Los fundamentos curriculares, los enfoques pedagógicos y didácticos con que se asume la organización educativa influyen directamente en la integración de las TIC, respaldado todo este proceso por la formación pertinente de los agentes educativos y el desarrollo de capacidades en la utilización de las herramientas tecnológicas en las acciones específicas de enseñanza y aprendizaje.

\section{B. Competencias y TIC}

El desarrollo de competencias es una exigencia en la educación actual, no solo para fomentarlas en los estudiantes, sino que son una responsabilidad personal y profesional en los docentes. Las competencias docentes están relacionadas directamente con el diseño de estrategias y acciones que susciten aprendizaje significativo, en las que el actor principal es el estudiante $\mathrm{y}$ en que las TIC entran a impulsar los respectivos procesos educativos en sintonía con sus realidades y necesidades [13].

"En el ámbito académico, la formación digital y el conocimiento pedagógico sobre el uso de las TIC permiten a los docentes mejorar su práctica pedagógica y apoyan simultáneamente la adquisición de competencias por parte de los estudiantes [13].". El maestro es "el responsable de diseñar tanto las oportunidades de aprendizaje como el entorno propicio en el aula que faciliten el uso de las TIC por parte de los estudiantes para aprender y comunicar" [14], por lo que deben prepararse para desempeñarse a cabalidad. En la actualidad esa capacidad con relación a las TIC, permiten guiar a los estudiantes en el desarrollo de competencias que están en sintonía con la sociedad del conocimiento, como son la reflexión crítica innovadora, aptitudes de colaboración y de tipo socioemocional, además de la resolución de problemas [15].

\section{Teorías educativas y uso de TIC}

Las TIC tienen la posibilidad de integrarse desde cualquier enfoque educativo, desde la esencia de cada uno de ellos. Desde modelo tradicional aunque no se considere indispensable el uso de materiales didácticos, las tecnologías cuando son utilizadas, solo entran a apoyar la función de transmisión de los saberes, contenidos técnicos y normas aceptadas cultural y socialmente [16]. En este caso, que es muy común en las instituciones educativas, las TIC solo entran a reforzar la pedagogía magistral y expositiva en donde el maestro tiene todo el dominio y el estudiante recepciona $y$ memoriza para aprender.

Los enfoques conductistas se evidencian en los programas educativos, desde su visión jerárquica y la organización del conocimiento. Las actividades para el aprendizaje se realizan respondiendo a objetivos de tipo instruccional, aplicando el refuerzo y el control para responder a objetivos terminales, asociados al cambio de conducta, se evalúa la acumulación de conocimientos de manera secuencial a través de exámenes cuya valoración es de tipo cuantitativo y se consolida de manera sumativa. El aprendizaje mediado por las TIC en este enfoque busca por ejemplo encontrar respuestas a estímulos que se presentan a través de programas, plataformas o pruebas estandarizadas, a las que se relacionan con refuerzos audiovisuales [17].

En [18] se asegura, que en el cognitivismo las TIC se aprovechan cuando involucran "comunicación sincrónica más que asincrónica en la interacción que se lleva a cabo entre el docente y sus alumnos y entre estos últimos. Así pues, un uso intensivo e interactivo de las TIC aumenta el nivel de aprendizaje". Ya que la interacción con herramientas docentes es importantes en el proceso de enseñanza y aprendizaje de los estudiantes, se pueden incorporar software que faciliten el diseño de mapas conceptuales y mentales, hipertextos, la utilización del internet para la exploración. Los recursos tecnológicos se incorporan teniendo en cuenta el pensamiento y los procesos internos de cada uno de los estudiantes, el procesamiento de la información y la consolidación de un nuevo conocimiento; la formación de conceptos, la solución de problemas, el lenguaje y adicionalmente posibilitando la participación activa en el proceso educativo y evaluativo.

El enfoque constructivista tiene en cuenta que la actividad y los procedimientos son fundamentales para generar el conflicto cognitivo, el descubrimiento y la construcción del conocimiento. El maestro como modelo y guía promueve actividades, desde la exploración y resolución de problemas, involucrando las tecnologías en contextos enriquecidos, teniendo en cuenta que a partir de los conocimientos previos se generan aprendizajes nuevos y productos de uso real. Las TIC en el marco del constructivismo debe ayudar a los estudiantes a pensar, a ser conscientes de sus procesos $\mathrm{y}$ 
estrategias mentales para mejorar el aprendizaje [18]. Las TIC "crean una experiencia diferente en el proceso de aprendizaje entre los estudiantes, se vinculan con la forma en la que ellos aprenden mejor, y funcionan como elementos importantes para la construcción de su propio conocimiento [19]".

Paralelamente el socio constructivismo facilita el aprovechamiento de las TIC en las diferentes modalidades educativas, ya que tienen un potencial significativo para posibilitar la interacción social y el trabajo colaborativo y cooperativo, que es sustancial en los avances en el aprendizaje y la construcción del conocimiento. Todas las influencias que se presentan a nivel social por intermedio de diferentes ambientes como la familia, las amistades, el ámbito laboral, inciden de una u otra forma en los procesos de formación personal y son tenidas en cuenta bajo la mirada del socio constructivismo. A partir de los postulados de Vygotsky se tiene en cuenta que los conocimientos se constituyen desde los propios esquemas de la persona en su contexto y su confrontación con los esquemas de las demás personas con los que se interactúa y el lenguaje es sustancial en este proceso [18].

Por tanto, con la utilización de las TIC desde la teoría educativa del socio constructivismo debe potenciarse la interacción con lo que es esencialmente comunitario y que hace parte de la realidad del estudiante, buscando solución a las problemáticas desde la participación grupal y la creación de espacios sociales, además de la consolidación de comunidades de aprendizaje. De allí el diseño de herramientas tecnológicas que enfatizan la comunicación, el intercambio de información y el trabajo colaborativo (software social). No se pierde de vista el desarrollo de los estudiantes desde el nivel real al potencial con el apoyo de los demás y del mismo docente como guía, favorecedor de saberes socioculturales y gestor de entornos de aprendizaje con pluralidad de instrumentos y recursos informativos [18].

En la actualidad se viene hablando del conectivismo "una teoría del aprendizaje para la era digital y globalizante", que algunos críticos la consideran más bien como una perspectiva pedagógica, la cual explica el aprendizaje complejo, centrando el desarrollo del conocimiento en la recursividad de la era tecnológica, superando lo meramente individual y evolucionando a la interacción del individuo con el mundo social digital que se encuentra en ágil evolución. Se consolida con la integración de las teorías de caos, redes, complejidad y auto-organización, busca la conexión de información especializada y se concentra en las conexiones que permiten un mayor aprendizaje. Desde el conectivismo las decisiones se fundamentan en principios cambiantes, por lo que distinguir entre la información importante y la que no lo es tanto es sustancial, además de la destreza de reconocer la capacidad de alteración que tiene la nueva información al entorno que ha sido influenciado por decisiones previas [20].

\section{Sociedad, educación y TIC}

Hay que tener presente que "la evolución de las tecnologías responde a los requerimientos de las relaciones sociales" [21]. Las TIC en función de la educación y no al contrario como se puede plantear desde una visión tecnocrática, se debe apuntar al desarrollo del pensamiento crítico, autónomo, creativo y en equipo. Las nuevas tecnologías permiten personalizar la formación de acuerdo a los intereses, habilidades y estilos de aprendizaje. El reto consiste en promover la creatividad y desarrollar en cada establecimiento educativo la capacidad de asumir y superar los diferentes desafíos y problemáticas particulares [22].

No hay que olvidar que "los sistemas educativos deben responder a los múltiples retos que les lanza la sociedad de la información, en función siempre de un enriquecimiento continuo de los conocimientos y del ejercicio de una ciudadanía adaptada a las exigencias de nuestra época [23]". En [24] se afirma que "La tecnología (o su carencia) plasma la capacidad de las sociedades para transformarse, así como los usos a los que esas sociedades, siempre en un proceso conflictivo, deciden dedicar su potencial tecnológico." Así mismo, "la tecnología no determina la sociedad. Tampoco la sociedad dicta el curso del cambio tecnológico, de modo que el resultado final depende de un complejo modelo de interacción".

\section{METODOLOGÍA}

Se realizó una investigación bajo un enfoque cualitativo, complementado con técnicas y análisis de información cuantitativa [25]. Se hizo un estudio de caso, donde se analizaron las creencias, formación, experiencia y competencias de los docentes de educación media o secundaria alta, desde el punto de vista de los mismos docentes, estudiantes respectivos, directivos docentes y padres de familia de estos estudiantes.

\section{A. Muestra de informantes}

La muestra de informantes la constituyeron miembros de la comunidad educativa de dos instituciones educativas del caribe colombiano A y B, estuvo conformada por 270 estudiantes (205 de la institución A y 65 de la B), 24 docentes (14 y 10), 5 directivos entre coordinadores y rectores (3 y 2), por último, 7 acudientes (5 y 2), para un consolidado total de 306 encuestados.

La muestra es de tipo no probabilística, con selección intencional, utilizando como criterio de inclusión la participación voluntaria. Para el caso de los tres primeros estamentos fue propuesta a todos sus miembros y para los padres o acudientes se dirigió a sus representantes ante las comisiones de evaluación en cada institución educativa.

Para las entrevistas de grupos focales, el número referente de entrevistados se definió teniendo en cuenta el número de directivos en cada institución educativa, 3 para la institución A y 2 para la institución B. Los docentes, estudiantes y padres de familia del nivel de educación media o secundaria alta se seleccionaron aleatoriamente.

\section{B. Instrumentos y técnicas para recoger la información.}

Para recoger la información se utilizaron diferentes técnicas: cuestionarios escala tipo Liker para las encuestas y entrevista semiestructurada a grupos focales de todos los estamentos. Los cuestionarios de las encuestas se diseñaron con 113 ítems, relacionados con las diez subcategorías y tres categorías de análisis, mientras que las entrevistas con 12 preguntas, igualmente discriminados por cada categoría y subcategoría. Ambos instrumentos fueron validados por expertos, cuyas opiniones permitieron ajustar el diseño en 
cuanto a su forma y contenido. La estructura de las entrevistas semiestructuradas aplicadas a los grupos focales de docentes, estudiantes, directivos y padres de familia conformados en cada institución, correspondían a las categorías y subcategorías de análisis de los cuestionarios, las cuales, respondieron las preguntas respectivas, propiciando la puesta en común de sus puntos de vista y de las percepciones que se tienen con respecto a la incorporación y apropiación de las TIC en los procesos de enseñanza y aprendizaje en la educación media o secundaria alta.

A continuación, se presenta en la Tabla I una caracterización de los cuestionarios aplicados, que incluye, el número de ítems para las encuestas, distribuidas por categorías y subcategorías de análisis, así:

TABLA I

NÚMERo De ÍTEMS DE LAS ENCUESTAS

\begin{tabular}{clc}
\hline \hline CATEGORIAS & \multicolumn{1}{c}{ SUBCATEGORIAS } & ITEMS \\
\hline \multirow{2}{\text{Incorporacióny}}{$\begin{array}{l}\text { Creencias de los Docentes. } \\
\text { Apropiación de TIC }\end{array}$} & $\begin{array}{l}\text { Formación y capacitación en el uso } \\
\text { de herramientas } \\
\text { Momentos o Niveles de } \\
\text { competencias }\end{array}$ & 14 \\
Enseñanza y Aprendizaje & $\begin{array}{l}\text { Estrategias de enseñanza y } \\
\text { aprendizaje }\end{array}$ & 20 \\
& Uso de TIC en la Práctica Docente. & 12 \\
Competencias TIC para & Competencia Tecnológica & \\
la Profesionalización & Competencia Comunicativa & 9 \\
Docente & $\begin{array}{l}\text { Competencia Pedagógica } \\
\text { Competencia De Gestión }\end{array}$ & 9 \\
& Competencia Investigativa & 9 \\
\hline \hline
\end{tabular}

Seguido se muestra en la Tabla II, el número de ítems de las entrevistas semiestructuradas, igualmente discriminados por cada categoría y subcategoría, como sigue:

TABLA II

NÚMERo De ÍTEMS DE LAS ENTREVISTAS SEMIESTRUCTURADAS

\begin{tabular}{clc}
\hline \hline CATEGORIAS & \multicolumn{1}{c}{ SUBCATEGORIAS } & ITEMS \\
\hline $\begin{array}{c}\text { Incorporación y } \\
\text { Apropiación de TIC }\end{array}$ & $\begin{array}{l}\text { Formación y capacitación en el } \\
\text { uso de herramientas } \\
\text { Momentos o Niveles de } \\
\text { competencias }\end{array}$ & 1 \\
$\begin{array}{c}\text { Enseñanza y } \\
\text { Aprendizaje }\end{array}$ & $\begin{array}{l}\text { Estrategias de enseñanza y } \\
\text { aprendizaje } \\
\text { Uso de TIC en la Práctica }\end{array}$ & 1 \\
$\begin{array}{l}\text { Docente. } \\
\text { Competencias TIC para } \\
\text { la Profesionalización } \\
\text { Docente }\end{array}$ & $\begin{array}{l}\text { Competencia Comunicativa } \\
\text { Competencia Pedagógica }\end{array}$ & 1 \\
& Competencia De Gestión & 2 \\
& Competencia Investigativa & 1 \\
\hline \hline
\end{tabular}

\section{Procesamiento de la información}

Para el análisis de la información cuantitativa, se utilizó el software estadístico SPSS. Se realizó un análisis de correspondencia múltiple, donde se calcularon tablas de contingencia utilizando el índice Chi cuadrado de Pearson $\left(\chi^{2}\right)$ como coeficiente de prueba. Se midió la significancia de las asociaciones de los estamentos encuestados, establecimientos educativos, género, edad y tiempo de utilización de las TIC con relación a los constructos definidos para incorporación y apropiación de TIC, enseñanza y aprendizaje y competencias TIC para la profesionalización docente.

A partir de los resultados promedios calculados con las respuestas cuantificadas en cada subcategoría y categoría, se llevó a cabo el mismo procedimiento para identificar las asociaciones estadísticamente significativas en cada una de ellas, con relación a los mismos aspectos (Estamentos encuestados, Establecimientos Educativos, Género, Edad y Tiempo de Utilización de las TIC).

Se hizo análisis de contenido aprovechando el software Atlas.ti. En el análisis de la información se procedió con la reducción de los datos, con la codificación y categorización de unidades, se establecieron relaciones y redes, haciendo el análisis respectivo frente a las bases teóricas que fundamentan la investigación.

\section{Fiabilidad y validez del instrumento de medida}

Se hizo un análisis de fiabilidad para medir la confiabilidad del instrumento. Para ello se utilizó el índice Alfa de Cronbach como coeficiente de prueba. Este es un coeficiente que produce valores que oscilan entre 0 y 1 , en donde valores próximos a 1 indican una alta confiabilidad y valores cercanos a cero, confiabilidad baja. Los valores del Alfa de Cronbach mostrados en la tabla 3, muestran que el instrumento tiene una buena consistencia interna [26].

La Tabla III que se presenta a continuación, refleja el análisis de fiabilidad a partir del Alfa de Cronbach:

TABLA III

ANÁlisis De Fiabilidad Del InSTRUMENTO Y Los Coeficientes Alfa De Cronbach, Utilizados Como Coeficiente De Prueba

\begin{tabular}{|c|c|c|c|}
\hline \multicolumn{2}{|c|}{ CONSTRUCTO } & $\begin{array}{c}\text { ALFA DE } \\
\text { CRONBACH }\end{array}$ & $\begin{array}{c}\text { No DE } \\
\text { ELEMENTOS }\end{array}$ \\
\hline \multirow{7}{*}{$\begin{array}{l}\text { Incorporación y } \\
\text { Apropiación de } \\
\text { TIC }\end{array}$} & Creencias De Los & 0,824 & 10 \\
\hline & Docentes. & & \\
\hline & Formación y & 0,843 & 14 \\
\hline & capacitación en el & & \\
\hline & $\begin{array}{l}\text { uso de } \\
\text { herramientas }\end{array}$ & & \\
\hline & Momentos o & 0,795 & 12 \\
\hline & $\begin{array}{l}\text { Niveles de } \\
\text { competencias }\end{array}$ & & \\
\hline \multirow{5}{*}{$\begin{array}{l}\text { Enseñanza y } \\
\text { Aprendizaje }\end{array}$} & Estrategias de & 0,816 & 20 \\
\hline & enseñanza y & & \\
\hline & aprendizaje & & \\
\hline & Uso de TIC en la & 0,862 & 12 \\
\hline & Práctica Docente. & & \\
\hline \multirow{5}{*}{$\begin{array}{l}\text { Competencias } \\
\text { TIC para la } \\
\text { Profesionalización } \\
\text { Docente }\end{array}$} & Competencia & & \\
\hline & Tecnológica & 0,871 & 9 \\
\hline & Competencia & & \\
\hline & Comunicativa & 0,836 & 9 \\
\hline & Pedagógica & 0,851 & 9 \\
\hline
\end{tabular}


Competencia De

Gestión

Competencia

Investigativa
0,829

0,842
9

9

Además, se hizo un análisis factorial confirmatorio para analizar la validez de constructo del instrumento, para lo que se utilizó el KMO como coeficiente de prueba [26]. El KMO, toma valores entre 0 y 1 , si $\mathrm{KMO} \geq 0,75$ la idea de realizar un análisis factorial es buena, si 0,75> KMO $\geq 0,5$ la idea es aceptable y si KMO < 0,5 es inaceptable. Este análisis del comportamiento psicométrico de las características de las variables, evidencia que éstas en su conjunto representan adecuadamente el concepto estudiado $(\mathrm{KMO}=0.827, \mathrm{P}$ $<0.05)$.

\section{RESULTADOS}

Para la categoría Incorporación y Apropiación, evidencia una homogeneidad de los resultados para cuatro de sus variables, es decir, se encontraron acuerdos estadísticamente significativos para Estamentos $\left(\chi^{2}=27,915 \mathrm{P}<0,05\right)$, Establecimientos Educativos $\left(\chi^{2}=19,342 \mathrm{P}<0,05\right)$, Edad $\left(\chi^{2}\right.$ $=36,547 \mathrm{P}<0,05)$ y Tiempo de Utilización de TIC $\left(\chi^{2}=\right.$ 43,979 $\mathrm{P}<0,059)$, que indican, que la incorporación $\mathrm{y}$ apropiación es moderada, dado que el $59.5 \%$ de los encuestados opinan que el nivel de incorporación y apropiación de las TIC por parte de los docentes no es algo frecuente, es decir las usan ocasionalmente.

La variable que más representatividad tiene es la de Establecimientos Educativos, en el sentido que todas las subcategorías evidencian significancia estadística, le sigue la variable Tiempo de Utilización de las TIC con dos subcategorías, por último, en un mismo nivel, Estamento y edad con solo una subcategoría. Esto indica, un mayor grado de homogeneidad en la variable de Establecimientos Educativos, que permite priorizar y profundizar el análisis desde esta perspectiva.

En la tabla IV y Fig. 1 se presenta la tabla cruzada y el diagrama de barras para la variable Establecimientos Educativos con respecto a la Incorporación y Apropiación de las TIC:

TABLA IV

DisTRIBUCIÓN DE RESPUESTAS POR ESTABLECIMIENTO EDUCATIVO CON RELACIÓN A INCORPORACIÓN Y APROPIACIÓN DE TIC

\begin{tabular}{|c|c|c|c|c|c|c|c|c|}
\hline & & & $\begin{array}{c}\text { Nun } \\
\text { ca }\end{array}$ & $\begin{array}{c}\text { Casi } \\
\text { nun- } \\
\text { ca }\end{array}$ & $\begin{array}{c}\text { A } \\
\text { veces }\end{array}$ & $\begin{array}{c}\text { Casi } \\
\text { siempre }\end{array}$ & $\begin{array}{l}\text { Siem- } \\
\text { pre }\end{array}$ & Total \\
\hline \multirow{4}{*}{$\begin{array}{l}\text { Esta- } \\
\text { bleci- } \\
\text { miento } \\
\text { educa- } \\
\text { tivo }\end{array}$} & \multirow[t]{2}{*}{$\begin{array}{l}\text { Institu- } \\
\text { ción A }\end{array}$} & $\begin{array}{l}\text { Recu- } \\
\text { ento }\end{array}$ & 0 & 24 & 145 & 58 & 0 & 227 \\
\hline & & $\begin{array}{l}\% \text { del } \\
\text { total }\end{array}$ & $\begin{array}{c}0,0 \\
\%\end{array}$ & $7,8 \%$ & $\begin{array}{c}47,4 \\
\%\end{array}$ & $19,0 \%$ & $0,0 \%$ & $\begin{array}{c}74,2 \\
\%\end{array}$ \\
\hline & \multirow[t]{2}{*}{$\begin{array}{l}\text { Institu- } \\
\text { ción B }\end{array}$} & $\begin{array}{l}\text { Recu- } \\
\text { ento }\end{array}$ & 2 & 6 & 37 & 32 & 2 & 79 \\
\hline & & $\begin{array}{l}\% \text { del } \\
\text { total }\end{array}$ & $\begin{array}{c}0,7 \\
\%\end{array}$ & $2,0 \%$ & $\begin{array}{c}12,1 \\
\%\end{array}$ & $10,5 \%$ & $0,7 \%$ & $\begin{array}{c}25,8 \\
\%\end{array}$ \\
\hline \multicolumn{2}{|c|}{ TOTAL } & $\begin{array}{l}\text { Recu- } \\
\text { ento }\end{array}$ & 2 & 30 & 182 & 90 & 2 & 306 \\
\hline
\end{tabular}

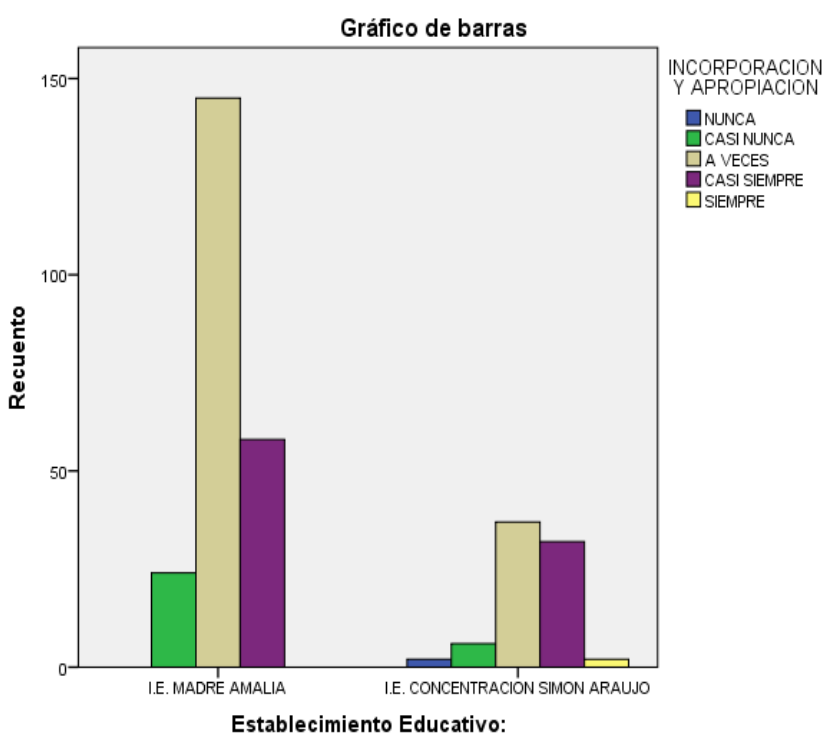

Fig. 1. Diagrama de barras de la categoría Incorporación y Apropiación de TIC en las instituciones educativas A y B.

A partir de los acuerdos estadísticamente significativos en los diferentes constructos que soportan las tres categorías de análisis, se focalizó el respectivo análisis detallado de los resultados obtenidos.

Desde la creencia de los docentes, se destaca la opinión de que, en algunas ocasiones, las TIC mejoran el ambiente en el aula de clases. En este sentido, sobresale la concepción favorable que existe en el $32.68 \%$ de los encuestados, quienes reconocen la importancia que los docentes le dan a las tecnologías en el mejoramiento del clima escolar en el aula de clases, lo que evidencia en cierto grado, el proceso de apertura que ha venido teniendo la incorporación de las TIC en el ámbito educativo de los establecimientos investigados $\left(\chi^{2}=\right.$ $11,994 \mathrm{P}<0,05)$.

Por otra parte, en los establecimientos educativos el 47,7\% de los docentes ocasionalmente han recibido orientación en el uso de herramientas $\left(\chi^{2}=9,639 \mathrm{P}<0,05\right)$ y este tipo de formación y capacitación en TIC se presenta desde el compromiso personal de actualización, lo que más se destaca entre las capacitaciones recibidas, se relaciona con el uso de herramientas para hacer presentaciones, procesar textos, creación y utilización de videos educativos en internet y el manejo básico de tabletas.

Con respecto a los momentos o niveles de competencia en los que se encuentran los docentes en el uso de las TIC, se identifica una percepción medianamente positiva $(55,9 \%)$, el $31,4 \%$ de los encuestados reconocen los avances en los niveles de competencias en algunos docentes $\left(\chi^{2}=17,220 \mathrm{P}<0,05\right)$. La mayoría de docentes de ambas instituciones se encuentran en niveles de exploración, algunos han avanzado a niveles de integración y excepcionalmente muy pocos a la innovación. 
A continuación, se muestra la Fig. 2 con la red de relaciones realizadas a partir del análisis de contenido de la categoría Incorporación y Apropiación de TIC en las instituciones educativas A y B:

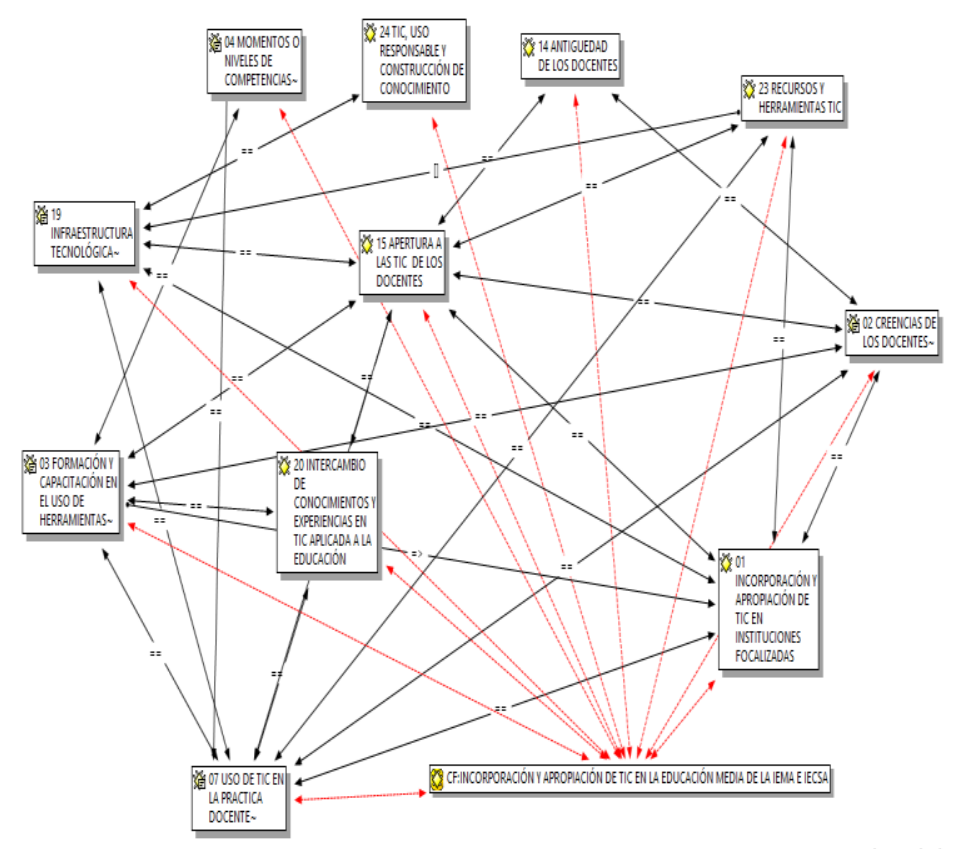

Fig. 2. Red de relaciones en la categoría Incorporación y Apropiación de TIC en las instituciones educativas A y B.

En cuanto a la categoría Enseñanza y Aprendizaje, presenta acuerdos estadísticamente significativos, particularmente en las variables de Establecimientos Educativos $\left(\chi^{2}=11,879 \mathrm{P}<\right.$ $0,05)$ y Tiempo de Utilización de las TIC $\left(\chi^{2}=44,185 \mathrm{P}<\right.$ $0,05)$. El $63.4 \%$ considera que las TIC en la práctica han sido medianamente útiles para fortalecer los procesos formativos, mientras que el $28.1 \%$ consideran que la experiencia demuestra que son de mucha o de suma importancia en las instituciones investigadas.

El resultado más sobresaliente está referido a la variable Establecimientos Educativos, en el sentido que la asociación estadística es significativa para ambas subcategorías $\left(\chi^{2}=\right.$ $\left.11,110 \mathrm{P}<0,05 ; \chi^{2}=14,358 \mathrm{P}<0,05\right)$.

En la tabla V y Figura 3 se presenta la tabla cruzada y el diagrama de barras para la variable Establecimientos Educativos con respecto a la categoría Enseñanza y Aprendizaje:

TABLA V

DISTRIBUCIÓN DE RESPUESTAS POR ESTABLECIMIENTO EDUCATIVO CON RELACIÓN A ENSEÑANZA Y APRENDIZAJE

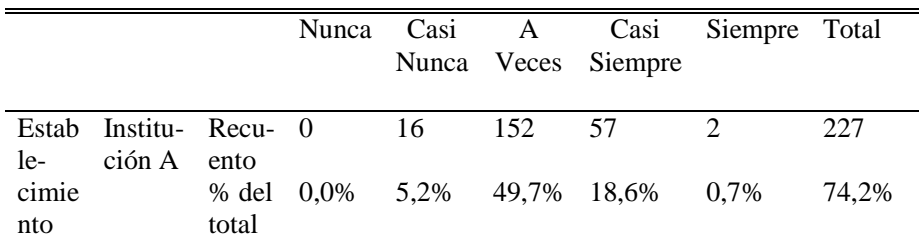

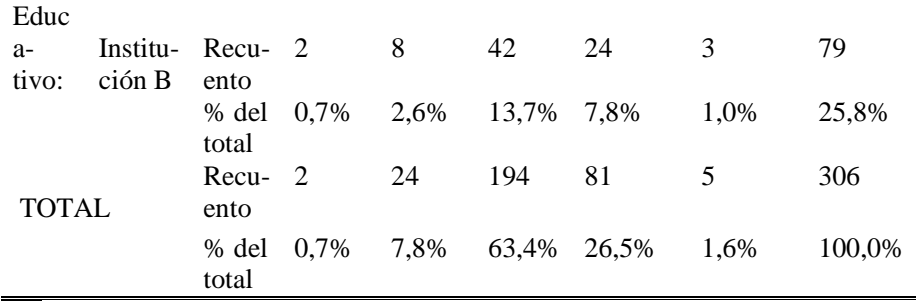

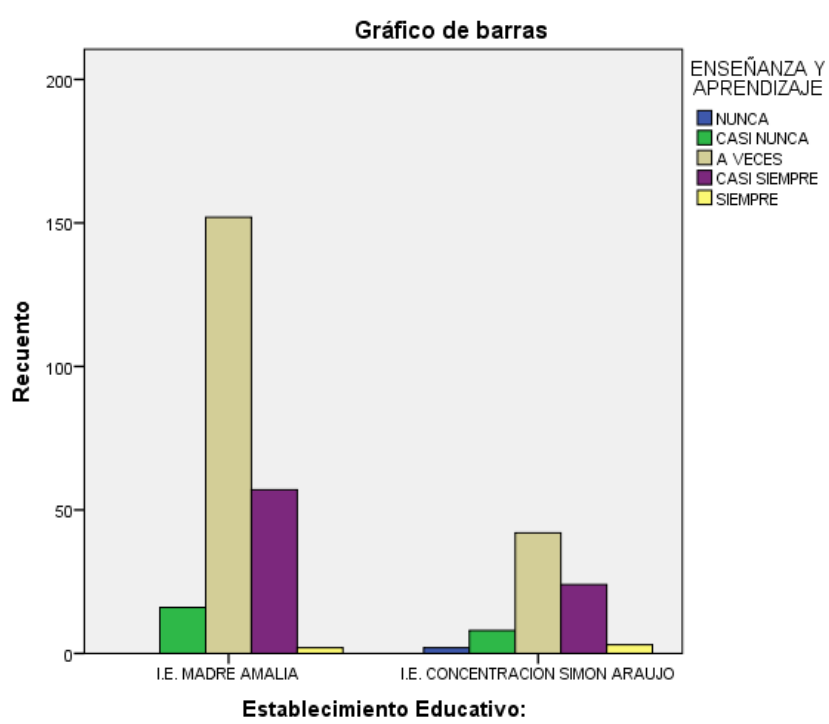

Fig. 3. Diagrama de barras de la categoría Enseñanza y Aprendizaje en las instituciones educativas A y B.

En la subcategoría de Estrategias de Enseñanza y Aprendizaje, se logra identificar un ocasional uso de las TIC por parte de los docentes en el proceso de enseñanza y aprendizaje, por lo que los estudiantes colaboran y cooperan mínimamente en esfuerzos de equipo, diferenciando e integrando redes de aprendizaje $\left(\chi^{2}=11,128 \mathrm{P}<0,05\right)$; además de que se realiza puntual y esporádicamente con decisiones sistemáticas y rigurosas, apoyadas por los medios tecnológicos más pertinentes $\left(\chi^{2}=9,851 \mathrm{P}<0,05\right)$. De igual forma, se llevan a cabo los siguientes aspectos: la interacción con ética y de manera apropiada $\left(\chi^{2}=11,401 \mathrm{P}<0,05\right)$, la identificación y solución de problemas concretos, la acción reflexiva y el enriquecimiento del conocimiento $\left(\chi^{2}=10,844\right.$ $\mathrm{P}<0,05)$, el desarrollo de tareas innovadoras realizadas eficientemente con los mejores resultados $\left(\chi^{2}=18,800 \mathrm{P}<\right.$ $0,05)$ y la comprensión del conocimiento técnico de las diversas tecnologías con un planteamiento interdisciplinario $\left(\chi^{2}=12,682 \mathrm{P}<0,05\right)$.

Es de anotar que en la medida que el tiempo de utilización de las TIC es mayor, la opinión tiende a inclinarse a ser más positiva. Se resalta que son más los que piensan que las generalizaciones a partir de la información recolectada y la formación integral utilizando TIC con competencia para comunicarse, conocer e investigar por sí mismos, se presenta por momentos.

Los resultados muestran una tendencia generalizada a considerar el empleo puntual, esporádico y excepcional de diferentes alternativas tecnológicas como: Blogs, Wikis, bases de datos, foros de discusión en Internet, Plataformas educativas (Moodle, Blackboard, etc.) y objetos virtuales de aprendizaje en el quehacer pedagógico. Por lo general no se 
crean ambientes innovadores con el uso de TIC, lo que se implementa es desde la proyección y consulta y desde una pedagogía tradicional. En este sentido, no hay acompañamiento sistemático en los procesos formativos con el apoyo de TIC o son pocos los educadores que lo hacen. Otra opinión que sobresale es lo que concierne a la insuficiente infraestructura tecnológica existente, incluyendo una conectividad insuficiente y con pocos equipos a disposición.

A continuación, se muestra la Fig. 4 con la red de relaciones realizadas a partir del análisis de contenido de la categoría Enseñanza y Aprendizaje en las instituciones educativas A y B

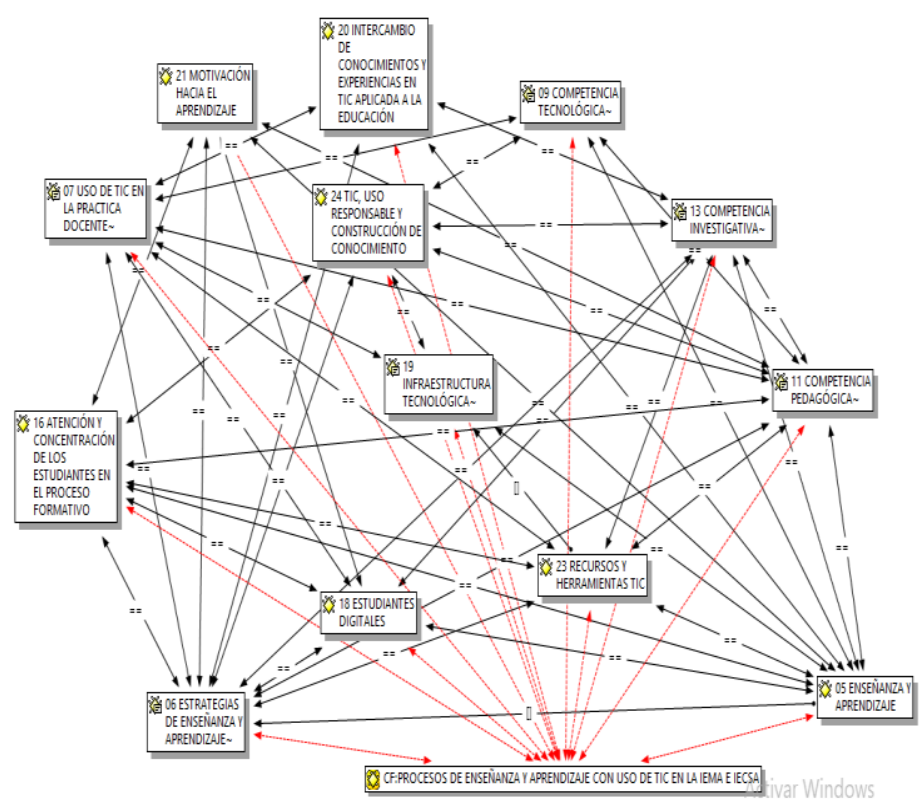

Fig. 4. Red de relaciones en la categoría Enseñanza y Aprendizaje con el uso de TIC en las instituciones educativas A y B.

Los resultados correspondientes a la categoria Competencias TIC para la profesionalización docente, presenta acuerdos estadísticamente significativos en 4 de las variables investigadas: Estamentos $\left(\chi^{2}=32,184 \mathrm{P}>0,05\right)$, Establecimientos Educativos $\left(\chi^{2}=15,997 \mathrm{P}>0,05\right)$, Edad $\left(\chi^{2}\right.$ $=44,102 \mathrm{P}>0,05)$ y Tiempo de Utilización de las TIC $\left(\chi^{2}=\right.$ $26,843 \mathrm{P}>0,05)$. A continuación, se refleja en la Tabla VI y en la Figura 5, el resultado tanto estadístico como grafico de la variable con más representatividad (Establecimientos Educativos), ya que el grado de significancia es respaldado por la homogeneidad detectada en cuatro de las cinco competencias TIC, consideradas claves para la profesionalización docente.

TABLA VI

ESTABLECIMIENTOS EDUCATIVOS CON RELACIÓN A COMPETENCIAS TIC PARA PROFESIONALIZACIÓN DOCENTE

\begin{tabular}{|c|c|c|c|c|c|c|c|c|}
\hline & & & $\begin{array}{l}\mathrm{Nu} \\
\text { nca }\end{array}$ & $\begin{array}{c}\text { Casi } \\
\text { Nunca }\end{array}$ & $\begin{array}{c}\text { A } \\
\text { Veces }\end{array}$ & $\begin{array}{c}\text { Casi } \\
\text { Siemp } \\
\text { re }\end{array}$ & $\begin{array}{l}\text { Siem- } \\
\text { pre }\end{array}$ & Total \\
\hline \multirow{3}{*}{$\begin{array}{l}\text { Estable- } \\
\text { Cimien- } \\
\text { to } \\
\text { Educa- } \\
\text { tivo: }\end{array}$} & \multirow{2}{*}{$\begin{array}{l}\text { Institu } \\
\text { ción A }\end{array}$} & $\begin{array}{l}\text { Recu- } \\
\text { ento }\end{array}$ & 1 & 37 & 155 & 34 & 0 & 227 \\
\hline & & $\begin{array}{l}\% \text { del } \\
\text { total }\end{array}$ & $\begin{array}{l}0,3 \\
\%\end{array}$ & $12,1 \%$ & $\begin{array}{l}50,7 \\
\%\end{array}$ & $\begin{array}{l}11,1 \\
\%\end{array}$ & $0,0 \%$ & $\begin{array}{l}74,2 \\
\%\end{array}$ \\
\hline & Institu & Recu- & 1 & 20 & 37 & 19 & 2 & 79 \\
\hline
\end{tabular}

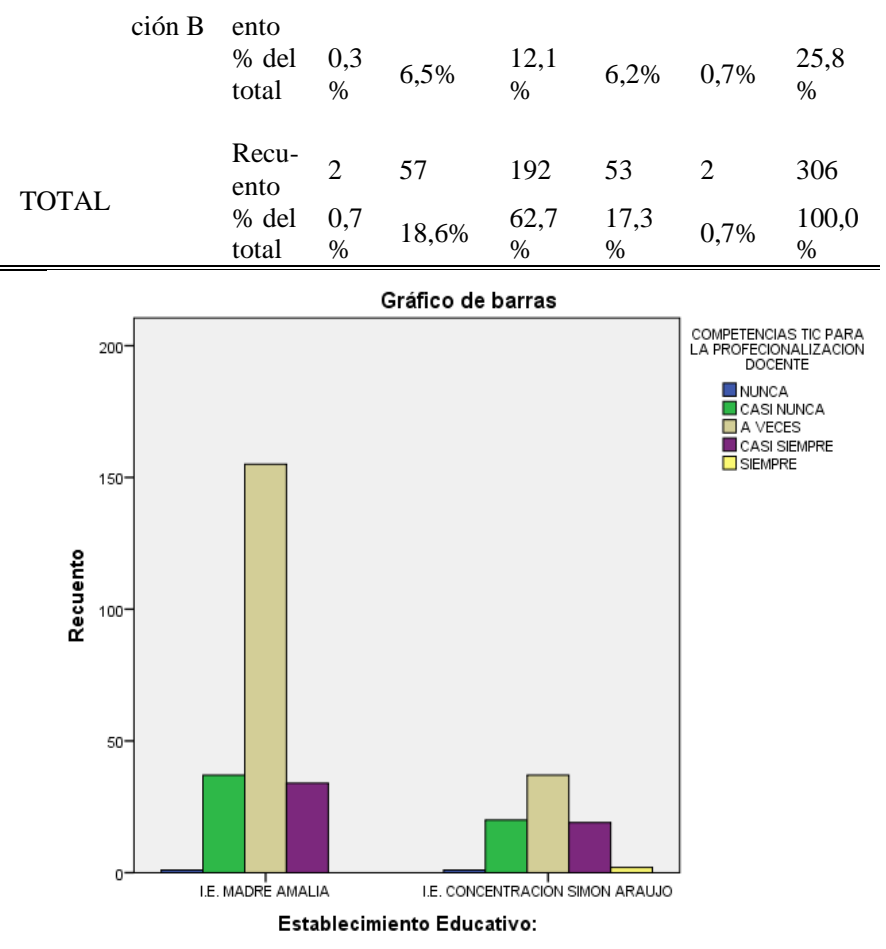

Fig. 5. Diagrama de barras de la categoría Competencias TIC para la Profesionalización Docente en las instituciones educativas A y B.

Se constata un mediano dominio de las Competencias TIC en el marco de la profesión docente $(62.7 \%)$. Se destacan levemente las competencias tecnológicas, comunicativa y de gestión, seguidas de la pedagógica e investigativa que se encuentran un nivel de desempeño inferior.

En la Competencia Tecnológica, en cuanto a la elaboración de actividades de aprendizaje utilizando aplicativos, contenidos, herramientas informáticas y medios audiovisuales $\left(\chi^{2}=11,268 \mathrm{P}<0,05\right)$, la consideración mayoritaria, es que los docentes lo hacen de manera esporádica $(57.27 \%$ en la Institución A y $43.04 \%$ en la Institución B). Existe una consideración favorable, con respecto a tener en cuenta los riesgos y potencialidades de publicar y compartir distintos tipos de información a través de Internet $\left(\chi^{2}=10,479 \mathrm{P}<\right.$ 0,05).

Por otra parte, la tendencia es hacia la mediana, poca o nula utilización de herramientas tecnológicas complejas o especializadas para diseñar ambientes virtuales de aprendizaje $\left(\chi^{2}=11,255 \mathrm{P}<0,05\right)$, que favorezcan el desarrollo de competencias en los estudiantes y la conformación de comunidades y/o redes de aprendizaje. El $47.06 \%$ tienen la postura de que esto se da en algunas ocasiones y el $32.35 \%$ concluyen que Nunca o Casi Nunca.

Dentro de la Competencia Tecnológica existe un resultado favorable en la utilización de herramientas tecnológicas para ayudar a los estudiantes a construir aprendizajes significativos y desarrollar pensamiento crítico $\left(\chi^{2}=12,918 \mathrm{P}<0,05\right)$ y la aplicación por parte de los docentes de las normas de propiedad intelectual y licenciamiento existentes, referentes al uso de información ajena y propia $\left(\chi^{2}=12,687 \mathrm{P}<0,05\right)$. En ambos constructos el comportamiento es muy similar, en la medida que hay una leve tendencia a tener en cuenta y aplicar estos aspectos en la práctica docente. 
En lo que tiene que ver con la Competencia Comunicativa, la navegación eficiente abordando la información no lineal, se presenta con regular frecuencia (39.87\%) en los establecimientos educativos $\mathrm{A}$ y $\mathrm{B}$, destacando un número importante de los que consideran que incluso es muy poco frecuente o que nunca se presenta $\left(\chi^{2}=16,650 \mathrm{P}<0,05\right)$. Existe una asociación estadísticamente significativa en las dos instituciones $\left(\chi^{2}=15,395 \mathrm{P}<0,05\right)$, en cuanto a la comunicación usando TIC de manera sincrónica y asincrónica, ya que la mayor parte considera que no se da frecuentemente o muy pocas veces $(42.16 \%)$, desarrollándose con limitaciones.

En el caso de la Competencia de Gestión (CG), se destacan aspectos como: la identificación de los elementos de la gestión escolar que pueden ser mejorados con el uso de las TIC, en las diferentes actividades institucionales $\left(\chi^{2}=14,920\right.$ $\mathrm{P}<0,05)$; las propuestas y el desarrollo de procesos de mejoramiento y seguimiento del uso de TIC en la gestión escolar $\left(\chi^{2}=21,380 \mathrm{P}<0,05\right)$; la adopción de políticas escolares existentes para el uso de las TIC que contemplan la privacidad, el impacto ambiental y la salud de los usuarios $\left(\chi^{2}\right.$ $=13,444 \mathrm{P}<0,05)$, por último, la dinamización de la formación de los colegas y la integración de las TIC de forma innovadora en sus prácticas pedagógicas $\left(\chi^{2}=21,869 \mathrm{P}<\right.$ 0,05). Para todos estos constructos, las respuestas de los encuestados en cada establecimiento educativo, se inclina en su mayoría, por considerar que la gestión de las TIC en función de la profesionalización de la práctica docente, se desarrolla ocasionalmente $(49.67 \%, 50 \%, 44.44 \%, 42.48 \%$, respectivamente).

Por su parte, en la Competencia Investigativa (CI) se destaca la documentación de observaciones del entorno y la práctica con el apoyo de TIC $\left(\chi^{2}=20,006 \mathrm{P}<0,05\right)$; la representación e interpretación de datos e información de investigaciones en diversos formatos digitales $\left(\chi^{2}=11,737 \mathrm{P}\right.$ $<0,05)$; al análisis con los estudiantes de la información proveniente de múltiples fuentes digitales $\left(\chi^{2}=17,350 \mathrm{P}<\right.$ $0,05)$ y la participación activa en redes y comunidades de práctica $\left(\chi^{2}=13,936 \mathrm{P}<0,05\right)$, para la construcción colectiva de conocimientos con estudiantes y colegas. Para todos estos constructos, las respuestas de los encuestados en cada establecimiento, se orientan a que la investigación con el apoyo de las TIC en función de la profesionalización de la práctica docente, se desarrolla por momentos $(46.7 \%, 43.1 \%$, $50.3 \%, 54.2 \%$ y $45.1 \%$, respectivamente).

La subcategoría Competencia Pedagógica (CP) evidencia un mayor grado de heterogeneidad en los resultados promedios ya que no se encontraron evidencias estadísticas de asociación en ninguna de las variables estudiadas. A pesar de ello, dentro de sus constructos, los que apuntan por una parte a incentivar en los estudiantes el aprendizaje autónomo y colaborativo, y por otra la evaluación de los resultados obtenidos con la implementación de estrategias haciendo uso de las TIC, promocionando una cultura de seguimiento, realimentación y mejoramiento permanente, reflejan una asociación estadísticamente significativa en la variable Establecimientos Educativos $\left(\chi^{2}=14,726 \mathrm{P}<0,05\right.$ y $\chi^{2}=$ $10,278 \mathrm{P}<0,05)$, aspectos que no se aplican de manera continua, sistemática y generalizada en las instituciones según los aportes correspondientes.
Las TIC son cada vez más utilizadas por los docentes en los procesos de gestión pedagógica e institucional con una integración básica; la débil infraestructura repercute en el desarrollo de la competencia tecnológica, además de sus creencias, formación, capacidades y destrezas necesarias para la apropiación e incorporación pertinente. Las experiencias de investigación están impulsadas principalmente por las exigencias que se presentan en los diferentes estudios de postgrado desarrollados por los docentes y directivos, por lo que son excepcionales los casos de docentes con reconocimiento investigativo; igualmente ocurre con las publicaciones que también se presentan en su mayoría en función de los estudios de postgrado y su correspondiente proceso de graduación.

Es de resaltar que se reconoce la importancia del uso de las TIC en la enseñanza y el aprendizaje en cuanto que estimula la actitud investigadora, pero se asocia en la práctica más que todo a los procesos de indagación, búsqueda y profundización de los conocimientos disciplinares asociados a cada una de las áreas orientadas por los docentes. La mayoría de docentes de ambas instituciones se encuentran en niveles de exploración, en cada una de las competencias TIC, algunos han avanzado a niveles de integración y excepcionalmente muy pocos a la innovación, en especial en el aspecto tecnológico, comunicativo y de gestión, seguidos de lo pedagógico e investigativo que se encuentran un nivel de desempeño inferior.

Seguidamente, se presenta la Fig. 6 con la red de relaciones elaboradas a partir del análisis de contenido de la categoría Competencias TIC para la Profesionalización Docente en las instituciones educativas A y B.

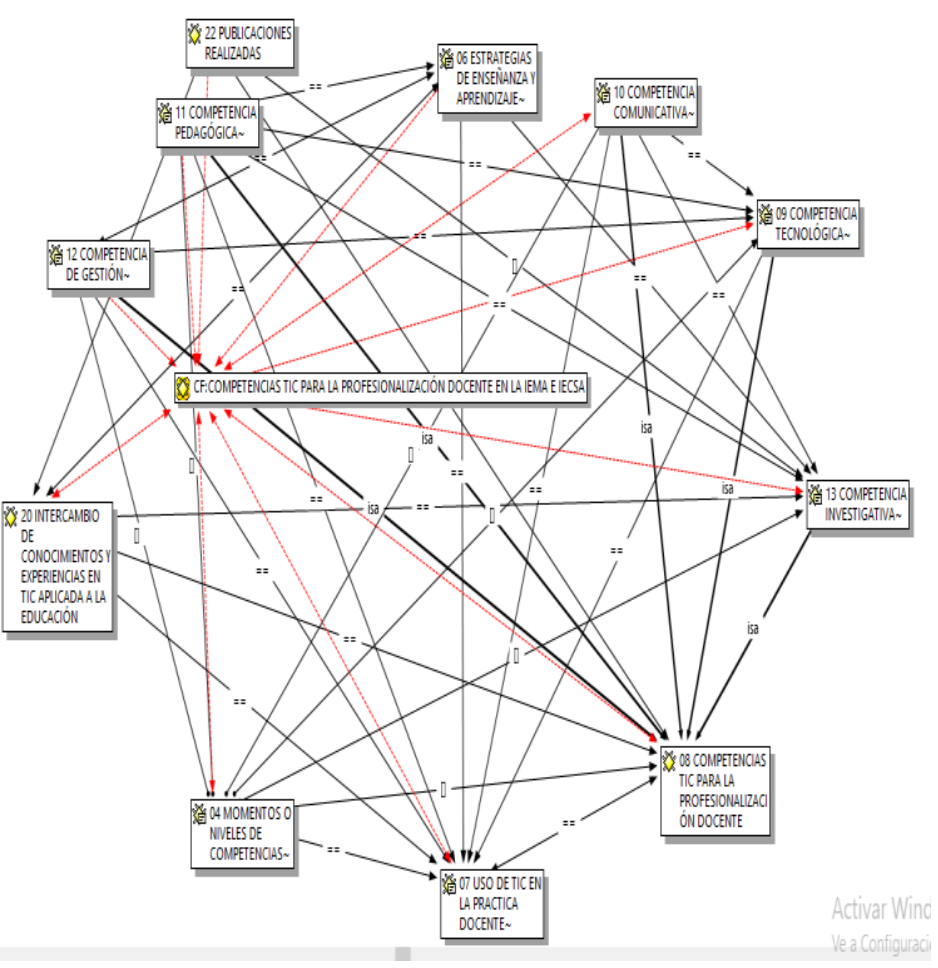

Fig. 6. Red de relaciones en la categoría Competencias TIC para la Profesionalización Docente en las instituciones educativas A y B. 


\section{CONCLUSIONES}

En cuanto a la incorporación y apropiación de las TIC se reconoce que no se da en forma permanente, debido a que solo algunos docentes consideran que su utilización mejora el ambiente de aula, motivan el aprendizaje y la producción escolar. Además, enfatizan que las capacitaciones recibidas están limitadas al manejo básico de tabletas, programas de ofimática y utilización de videos educativos en internet. Hay un reconocimiento en el avance de los niveles de competencias por parte de los docentes, ya que la mayoría pueden utilizar las TIC por sí mismos, asumiendo las implicaciones éticas, inculcando su uso responsable en la comunidad educativa. Se evidencia la necesidad de mejorar el aprovechamiento de herramientas comunicativas, específicamente en el uso del correo electrónico y en ambientes de aprendizaje que promuevan el trabajo colaborativo en el aula. Los docentes que tienen mayor nivel de competencias TIC son aquellos que en su formación tienen relación con el uso de las herramientas tecnológicas aplicadas en educación.

En general, hay una tendencia positiva a considerar relevante la utilización de las TIC en el proceso de enseñanza y aprendizaje, pero en la práctica se constata que la mayoría de los docentes las usan ocasionalmente para realizar: trabajo cooperativo, consolidación de redes de aprendizaje, aprendizaje basado en la identificación y resolución de problemas, las tareas innovadoras, la acción reflexiva y critica de cara a enriquecer el conocimiento. Las TIC son empleadas en su mayoría en un contexto pedagógico tradicional, como un recurso instrumental para trasmitir conocimiento, descuidando la formación integral de los estudiantes. Por lo tanto, las variadas herramientas que se encuentran al alcance en la actualidad, entre las que se encuentran: Blogs, Wikis, bases de datos, foros de discusión en Internet, plataformas educativas (Moodle, Blackboard, etc.) y objetos virtuales de aprendizaje son casi que ignoradas en las prácticas de aula que concretan los fundamentos pedagógicos, la acción didáctica y la planeación curricular asumida en las instituciones educativas. El correo electrónico está más en función de los procesos comunicativos institucionales que de los educacionales, por su parte el chat y las redes sociales es casi nula su adaptación a la labor formativa.

Existe para la mayoría de docentes, un divorcio entre la utilización de las TIC y el desarrollo del pensamiento crítico, además de no apuntar a la integralidad del desarrollo humano, entre otros aspectos, por su poca utilización y por ser introducidas en prácticas de tipo tradicional como un recurso adicional. Esto se contradice con la aceptación que se tiene de las TIC como aliados estratégicos para una mayor motivación, la construcción del conocimiento, interacción social, ejecución de proyectos contextualizados y el desarrollo de trabajos de investigación. Por otro lado, hay que tener en cuenta, que se presentan grandes dificultades en cuanto a conectividad, infraestructura y dotación tecnológica.

Las competencias TIC para la profesionalización docente presentan un mediano dominio en las instituciones investigadas, ubicándose en la mayoría de los docentes en un nivel de exploración, uno pocos en nivel de integración y casos excepcionales en el de innovación. Existe un regular desarrollo de la Competencia Tecnológica en los docentes, especialmente por el poco dominio que se presenta de aplicativos, contenidos, herramientas informáticas y medios audiovisuales en la enseñanza y aprendizaje de los estudiantes. Por tal motivo, no hay combinación de variadas herramientas tecnológicas para mejorar la planeación e implementación de las prácticas educativas por parte de los docentes. Se destaca a nivel de esta competencia lo que tiene que ver con la sensibilización que se hace de los riesgos y potencialidades de publicar y compartir distintos tipos de información a través de Internet, al igual que la mínima, pero creciente disposición de utilizar las herramientas tecnológicas para ayudar a los estudiantes a construir aprendizajes significativos.

En cuanto a la Competencia Comunicativa con el uso de las TIC, se lleva a cabo limitadamente tanto de forma sincrónica (al instante) como asincrónica (diferida en el tiempo), con informaciones de tipo general por lo que se identifica la necesidad de fortalecer la comunicación con estudiantes y sus familiares/acudientes, los colegas y la retroalimentación con grupos de investigación, desde la realidad de los procesos educacionales. Además, existe dificultad en la navegación eficiente en internet, integrando fragmentos de información presentados de forma no lineal; lo que se presenta igualmente con la representación de la información para ser utilizados con propósitos educativos; y en la contribución con conocimientos en repositorios virtuales, con textos diversos.

En el caso de la Competencia de Gestión, se presenta un regular desarrollo de los elementos de la gestión escolar que pueden ser mejorados con el uso de las TIC, entre los que se encuentran: las propuestas para de mejoramiento y seguimiento del uso de TIC en la gestión escolar; la adopción de políticas escolares para el adecuado uso de las TIC; por último, la dinamización de la formación de los colegas y la integración de las TIC de forma innovadora. Por su parte, en la Competencia Investigativa se utilizan de manera esporádica la documentación de observaciones del entorno y la práctica educativa con TIC, la representación e interpretación de la información en diversos formatos digitales; el análisis con los estudiantes de la información proveniente de fuentes virtuales, al igual que el fomento de la participación activa en redes de aprendizaje y práctica.

Por último, la Competencia Pedagógica, refleja poca frecuencia de uso de las TIC en el seguimiento, realimentación, evaluación y mejoramiento permanente de los procesos de enseñanza y aprendizaje, además del poco uso pertinente de las herramientas tecnológicas en las prácticas de aula, desde enfoques pedagógicos participativos, constructivos desde lo cognitivo y a partir de la interacción y transformación social de la realidad contextualizada.

Ante la situación identificada con relación al aprovechamiento de las TIC en la enseñanza y aprendizaje y las competencias docentes necesarias en las respectivas instituciones educativas, se hace indispensable desarrollar políticas y estrategias institucionales en diez líneas de acción para el mejoramiento, que se englobe en un Plan Tecnológico Institucional que contempla lo que a continuación se indica y que repercute no solo en la educación media, sino en todos los niveles educativos:

$\checkmark$ Apertura de espacios de dialogo y acuerdos institucionales en torno a la incorporación y apropiación de las TIC en el contexto educativo. 
$\checkmark \quad$ Incorporación y apropiación efectiva de las TIC en el PEI, el currículo y la práctica educativa.

$\checkmark$ Ampliación y mejoramiento de la infraestructura tecnológica que garantice el uso de las TIC en el desarrollo de las actividades escolares. (incluye aulas focalizadas con TIC).

$\checkmark$ Garantizar la conectividad adecuada y permanente, permitiendo no solo que la sala de informática, sino que las aulas cuenten con acceso a Internet.

$\checkmark \quad$ Impulso de una cultura comunicativa a través de TIC (creación y/o aprovechamiento pertinente de plataformas institucionales o páginas WEB- redes sociales).

$\checkmark \quad$ Capacitación permanente a los docentes y directivos en TIC, pedagogía y didáctica.

$\checkmark \quad$ Planear y realizar prácticas de aula apoyadas en TIC desde enfoques pedagógicos participativos, socio constructivistas, que posibiliten aprendizajes significativos y transformantes.

$\checkmark \quad$ Seguimiento permanente al uso de TIC en el aula.

$\checkmark$ Conformación y participación en redes virtuales de aprendizaje y práctica educativa.

$\checkmark \quad$ Fomentar y financiar procesos investigativos con apoyo de TIC y en TIC

\section{REFERENCIAS}

[1] Cumbre Mundial sobre la Sociedad de la Información, CMSI. Ginebra, Túnez. 2003, 2005. [En línea]. Disponible en http://www.itu.int/net/wsis/index-es.html.

[2] CEPAL, "Compromiso de San Salvador y plan de acción eLAC 2010", en Conferencia Ministerial sobre la Sociedad de la Información de América Latina y el Caribe, San Salvador, 2008, pp. 1-21. [En línea]. www.cepal.org/cgibin/getprod.asp?xml=/socinfo/noticias/paginas/6/32526/P32526.xml \&xsl=/socinfo/tpl/p18f

[3] Ministerio de Educación Nacional de Colombia, Competencias TIC para el desarrollo profesional docente. Bogotá: Oficina de Innovación Educativa con Uso de Nuevas Tecnologías MEN. 2013. pp.1-71. [En línea]. Disponible en https://www.mineducacion.gov.co.

[4] L. Escorcia Oyola y C. Jaimes de Triviño, "Tendencias de uso de las TIC en el contexto escolar a partir de las experiencias de los docentes", Educ. y Educ., vol. 18, no. 1, pp. 137-152, Jun. 2015. doi: 10.5294/edu.2015.18.1.8.

[5] A. M. Caicedo Tamayo y T. Rojas Ospina, "Creencias, conocimientos y usos de las TIC de los profesores universitarios", Educ. y Educ., vol. 17, no. 3, pp. 517-533, Dic. 2014. doi: 10.5294/edu.2014.17.3.7.

[6] A. Cortes Rincón, "Prácticas innovadoras de integración educativa de TIC que posibilitan el desarrollo profesional docente", tesis doctoral, Dpto Pedagogía Aplicada, Prog. Doctorado en Educación, Univ. Autónoma de Barcelona, Bogotá, 2016. pp. 1-313. [En línea]. Disponible

https://www.tdx.cat/documents/12/82/51/12825185841547165615151 9552371778603466/document_1.pdf

[7] P. Marqués Graells, "Impacto de las TIC en la educación: funciones y limitaciones", 3 Ciencias, vol. 1, no 3, pp.14-29, Dic. 2012. [En línea]. Disponible en https://www.3ciencias.com/revistas/revista/3ctic-no-3/

[8] L. Stojanovic Casas, "Tecnologías de comunicación e información en educación: referentes

para el análisis de entornos virtuales de enseñanza aprendizaje", Revista de Investigación, vol. 32, no 65, pp. 83-122, Dic. 2008. [En línea]. Disponible http://www.scielo.org.ve/scielo.php?script=sci_arttext\&pid=S101029142008000300006

[9] E. Blanco, C. Rico y M. Pino, "Utilización y funcionalidad de los recursos tecnológicos y de las nuevas tecnologías en la educación superior", Educação \& Sociedade, vol. 30, no 109, pp. 1209-1225, Sep. 2009. DOI:10.1590/S0101-73302009000400014

[10] R. Tirado Morueta y J. I. Aguaded Gómez, "Influencias de las creencias del profesorado sobre el uso de la tecnología en el aula", Revista de Educación, no. 363, pp. 230-255, Ene. 2014. doi: 10.4438/1988-592X-RE-2012-363-179

[11] A. Huertas Montes y A. Pantoja Vallejo, "Efectos de un programa educativo basado en el uso de las TIC sobre el rendimiento académico y la motivación del alumnado en la asignatura de Tecnología de Educación Secundaria”, Educación XX1, vol. 19, no. 2, PP. 229-250, May. 2016. DOI: 10.5944/educxx1.16464

[12] J. E. Padilla Beltrán, P. L. Vega Rojas y D. A. Rincón Caballero, "Tendencias y dificultades para el uso de las TIC en educación superior", Entramado, vol. 10, no. 1, pp. 272-295, Ene. 2014. [En línea]. Disponible

http://www.scielo.org.co/pdf/entra/v10n1/v10n1a17.pdf

[13] C. A. Hernández Suárez, M. A. Arévalo Duarte y A. A. Gamboa Suárez, "Competencias TIC para el desarrollo profesional docente en educación básica", Praxis \& Saber, vol. 7, no. 14, pp. 41 - 69, Jul. 2016. DOI: https://DOI.org/10.19053/22160159.5217

[14] UNESCO, Normas sobre competencia en TIC para docentes. Londres: Organización de las Naciones Unidas para la Educación, la Ciencia y la Cultura. 2008. pp. 1-28. [En línea]. Disponible en https://www.campuseducacion.com/blog/wpcontent/uploads/2017/02/Normas_UNESCO_sobre_Competencias_en TIC_para_Docentes.pdf

[15] UNESCO, Marco de competencias de los docentes en materia de TIC. París: Organización de las Naciones Unidas para la Educación, la Ciencia y la Cultura. 2019. pp. 1-64. [En línea]. Disponible en https://unesdoc.unesco.org/ark:/48223/pf0000371024?posInSet=1\&q ueryId=d10f8a52-7242-4801-aa5d-f03e7609453f

[16] J. De Subiría Samper, Los modelos pedagógicos. Hacia una pedagogía dialogante, 2a ed.. Bogotá:

Cooperativa Editorial Magisterio, 2006. [En línea]. Disponible en https://books.google.com.co/books?id=wyYnHpDT17AC\&printsec=c opyright\&hl=es\&redir_esc $=\mathrm{y} \# \mathrm{v}=$ onepage $\& \mathrm{q} \& \mathrm{f}=$ false

[17] G. Vergara Ríos y H. Cuentas Urdaneta, "Actual vigencia de los modelos pedagógicos en el contexto educativo". Serbiluz, vol. 31, no. 6, pp. 914-934, 2015. [En línea]. Disponible en https://dialnet.unirioja.es/descarga/articulo/5758752.pdf

[18] F. J. Valdez Alejandre, "Teorías educativas y su relación con las tecnologías de la información y de

la comunicación (TIC)", en XVII congreso internacional de Contaduría, Administración e Informática. Mexico D.C., 2012. [En línea]. Disponible congreso.investiga.fca.unam.mx/docs/xvii/docs/L13.pdf (2012).

[19] S. Hernández Requena, "El modelo constructivista con las nuevas tecnologías: aplicado en el proceso de aprendizaje", Revista de Universidad y Sociedad del Conocimiento, vol. 5, no. 2, pp. 26-35, Oct. 2008. [En línea]. Disponible en: http://www.redalyc.org/articulo.oa?id=78011201008

[20] G. Siemens, "Conectivismo: Una teoría de aprendizaje para la era digital", Dic. 2004. [En línea]. Disponible en: https://docs.google.com/document/d/1ZkuAzdx1191DgcC1E_XSmPTOk6Gu1K2SEvXtduG3gc/edit\#!

[21] J. C. Tedesco, Educar en la sociedad del conocimiento, Mexico D.F.: Fondo de

Cultura Económica, 2006. [En línea]. Disponible en: https://socioeducacion.files.wordpress.com/2011/05/tedesco-carloseducar-en-la-sociedad-del-conocimiento.pdf

[22] S. K. Robinson, Out of our minds. Learning to be creative. Chichester: Capstone Publishing Ltd., 2011.

[23] J. Delors et. al., La educación encierra un tesoro. Paris: Ediciones Santillana-UNESCO, 1996. [En línea]. Disponible en: https://unesdoc.unesco.org/ark:/48223/pf0000109590_spa

[24] M. Castells, La era de la información: economía, sociedad y cultura, 2a ed. Madrid: Alianza Editorial, 2000. [En línea]. Disponible en: https://books.google. com.co/books?id=uADgO-

fONJgC\&printsec $=$ frontcover\&hl=es\&source $=$ gbs_ge_summary_r\&c $\mathrm{ad}=0 \# \mathrm{v}=$ onepage $\& \mathrm{q} \& \mathrm{f}=$ false

[25] J. W. Creswell, Research design: qualitative, quantitative, and mixed methods approaches. 3a ed.. Thousand Oaks, SAGE Publications. Inc., 2009.

[26] J. García, M. González y B. Ballesteros. Introducción a la investigación en educación. Madrid: UNED. 2001 


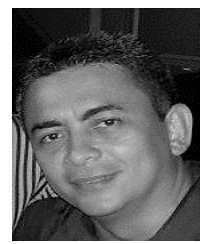

Robert Ricardo Alvarez Sampayo, Especialista en Docencia, Ingeniero Agrícola de la Universidad de Sucre, Directivo DocenteCoordinador de la Institución Educativa Madre Amalia del municipio de Sincelejo (Colombia), Maestrante VI Cohorte Maestría en Educación SUECARIBE - Universidad de Sucre. Experiencia laboral anterior, como asesor en Desarrollo Humano Rural en la Diakonía de la Paz (Diócesis de Sincelejo), docente de Ciencias Naturales y Educación Ambiental en Los Patos (Majagual - Sucre), Coordinador en la Institución Educativa Nuestra Señora del Rosario del municipio de San Antero (Córdoba).

ORCID: https://orcid.org/0000-0002-6258-5804

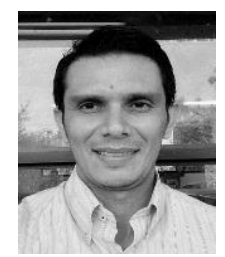

Ricardo Ramon Sarmiento Guevara, Especialista en Docencia, Ingeniero de Sistemas de la Fundación Universitaria San Martín, Docente de Tecnología e Informática de la Institución Educativa Concentración Simón Araujo del Municipio de Sincelejo (Colombia), Maestrante VI Cohorte Maestría en Educación SUECARIBE - Universidad de Sucre. Experiencia laboral anterior, como Docente de Tecnología e Informática en la Institución Educativa María Inmaculada (San Marcos, Sucre Colombia) e Instructor en la Caja de Compensación Familiar de Sucre COMFASUCRE (Sincelejo).

ORCID: https://orcid.org/0000-0001-9094-2867

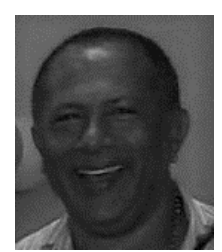

Tulio Rafael Amaya de Armas, Maestría en innovación e investigación en educación Uned, Doctor en investigación e investigación en didáctica Uned, Artículos: Evaluación del conocimiento de futuros profesores sobre las transformaciones de las representaciones de una función Revista Educación matemática, ISSN 165-5826, diciembre de 2016 Ciudad de México, México. Dificultades de los estudiantes de once grados al hacer transformaciones de representaciones de una función. Errores y dificultades que presentan los estudiantes de octavo grado al factorizar polinomios. Acta publicación anual CLAME julio de 2015 vol 28. Dificultad o fortaleza en el aprendizaje de los estudiantes en el trabajo con fracciones algebraicas Cap. 2 Clame julio 2015. Errores de los estudiantes en el trabajo pre-algebraico Análisis del discurso matemático escolar, en el Acta latinoamericana de Matemática Educativa, vol 24, 2011

ORCID: https://orcid.org/0000-0003-0342-4338 